\title{
Review Article \\ Recent Approaches for Broadening the Spectral Bandwidth in Resonant Cavity Optoelectronic Devices
}

\author{
Gun Wu Ju, ${ }^{1}$ Byung Hoon Na, ${ }^{2}$ Yong-Hwa Park, ${ }^{2}$ Young Min Song, ${ }^{3}$ and Yong Tak Lee \\ ${ }^{1}$ School of Information and Communications, Gwangju Institute of Science and Technology, Gwangju 500-712, Republic of Korea \\ ${ }^{2}$ Imaging Device Lab, Samsung Advanced Institute of Technology, Suwon-si 443-803, Republic of Korea \\ ${ }^{3}$ Department of Electronics Engineering, Pusan National University, Busan 609-735, Republic of Korea \\ ${ }^{4}$ Advanced Photonics Research Institute, Gwangju Institute of Science and Technology, Gwangju 500-712, Republic of Korea
}

Correspondence should be addressed to Young Min Song; ysong@pusan.ac.kr and Yong Tak Lee; ytlee@gist.ac.kr

Received 9 May 2015; Accepted 1 September 2015

Academic Editor: Rosa Lukaszew

Copyright (C) 2015 Gun Wu Ju et al. This is an open access article distributed under the Creative Commons Attribution License, which permits unrestricted use, distribution, and reproduction in any medium, provided the original work is properly cited.

\begin{abstract}
Resonant cavity optoelectronic devices, such as vertical cavity surface emitting lasers (VCSELs), resonant cavity enhanced photodetectors (RCEPDs), and electroabsorption modulators (EAMs), show improved performance over their predecessors by placing the active device structure inside a resonant cavity. The effect of the optical cavity, which allows wavelength selectivity and enhancement of the optical field due to resonance, allows the devices to be made thinner and therefore faster, while simultaneously increasing the quantum efficiency at the resonant wavelengths. However, the narrow spectral bandwidth significantly reduces operating tolerances, which leads to severe problems in applications such as optical communication, imaging, and biosensing. Recently, in order to overcome such drawbacks and/or to accomplish multiple functionalities, several approaches for broadening the spectral bandwidth in resonant cavity optoelectronic devices have been extensively studied. This paper reviews the recent progress in techniques for wide spectral bandwidth that include a coupled microcavity, asymmetric tandem quantum wells, and high index contrast distributed Bragg-reflectors. This review will describe design guidelines for specific devices together with experimental considerations in practical applications.
\end{abstract}

\section{Introduction}

Multilayer thin film growth and coatings have been investigated for many years as distributed Bragg-reflector (DBR) or Fabry-Perot cavities to produce resonant enhancement in numerous optoelectronic devices such as light emitting diodes [1], photodetectors [2], low-threshold lasers [3, 4], and modulators [5]. Recently, to achieve multiple functionalities, it is necessary to optimize parameters related to performance such as the spectral bandwidth, angular bandwidth, wavelength selectivity, and polarization. In particular, the wide spectral bandwidth characteristic of optoelectronic devices allows high manufacturing tolerance, wide angular properties, and robust operation. Several studies have demonstrated these characteristics using microcavity structures, asymmetric tandem quantum wells, and high index contrast DBR structures.
In wavelength division multiplexed (WDM) systems, it is desirable to have wavelength selective photodetectors with low channel crosstalk at the channel spacing $(<4 \mathrm{~nm})$ [6-8]. The resonant cavity enhanced photodetector (RCEPD) benefits from wavelength selectivity and a large increase of the resonant optical field introduced by the Fabry-Perot cavity. Thin absorption layers are inserted into a resonant cavity sandwiched by two mirrors, forming a Fabry-Perot cavity. This results in an increased optical field and therefore faster speed, while simultaneously increasing the quantum efficiency at the resonant wavelength [9]. Narrow spectral bandwidth can be achieved by adopting a high-Q cavity design in the conventional single-cavity RCEPD structure or by employing a narrow-band Fabry-Perot filter. However, it is very important to have a flat-topped passband when the channel-aligning tolerance between the transmitter and the receiver is considered, and thereby the system performance will not be sensitive 
to wavelength fluctuation in the transmitter or the resonant control in the receiver [10]. During the past two decades, numerous theoretical and experimental methods have been reported to address this crucial need [10-13]. The concept underlying the designed structures was realizing a low loss cavity for coupling with different mirrors such as compound semiconductors, dielectric materials, and Au metals.

Another application using a coupled cavity is an electroabsorption modulator (EAM) used as an optical shutter in a three-dimensional (3D) image capturing system based on time of flight. This is a simple and inexpensive method to realize a $3 \mathrm{D}$ image with a small system. The basic $3 \mathrm{D}$ imaging system consists of an active illumination light source as an optical transmitter and a demodulator and a detector as a receiver. The modulated light source travels toward the object, and the light reflected from the object reaches the detector. A 3D image of an object can be realized by extracting distance information from the phase delay between the illuminated light and reflected light, which is achieved using a large area optical shutter. To obtain high resolution depth image information, it is thus essential that the large area optical shutter has high performance such as high modulation depth [14] and high-speed modulation, which can increase the resolution depth [15]. High-speed modulation is obtained by utilizing the electroabsorption mechanism of a multilayer structure, which has an optical resonance cavity and light absorption in quantum wells. The optical shutter is positioned in front of a standard high resolution complementary metal oxide semiconductor image sensor. The optical shutter modulates the incoming infrared image to acquire the depth image [16]. Despite advances to improve the modulation speed and modulation depth, this approach still suffers from relatively narrow spectral bandwidth due to the high quality factor of the Fabry-Perot cavity. The narrow spectral bandwidth is also problematic since the modulation depth degrades significantly as the emission wavelength of the light source used in the time of flight based 3D imaging system varies according to temperature. Moreover, a narrow spectral bandwidth reduces the manufacturing tolerance of the 3D imaging system and the operating temperature range since the bandgap of a semiconductor material changes as the temperature changes. Thus, in order to improve the operational reliability of $3 \mathrm{D}$ imaging systems, the EAM should be capable of operating over a wide spectral bandwidth with high modulation depth.

High index contrast DBR structures are used in a wide range of applications such as vertical cavity surface emitting lasers (VCSELs), filtering, and wavelength division multiplexing. The reflectivity and spectral bandwidth directly depend on the refractive index contrast between the high and low index materials. Moreover, a higher index contrast guarantees higher tolerance during the fabrication process of the DBRs because the deposition rate dramatically affects the quality of the optical reflective wavelength, and highly tolerant DBRs are required for low-cost fabrication. Holonyak and coworkers have established a technique involving wet oxidation of AlAs [17-19] and have shown that AlAs can be oxidized without considerably affecting the GaAs/AlAs DBR system. Since the refractive index of oxidized AlAs is low (1.5), the combination of oxidized AlAs and GaAs shows favorable properties of broad spectral bandwidth [19]. Recently, it was shown that oblique-angle deposition (OAD) of materials, for example, $\mathrm{SiO}_{2}, \mathrm{TiO}_{2}$, ITO, and amorphous silicon (a-Si), can be used to fabricate DBR structures with optical properties that can be controlled by the oblique angle [20-22]. By controlling the angle of deposition and thereby the layer porosity and refractive index, OAD allows easy fabrication of a DBR composed of a single material chosen for its optical properties in which each layer has a different refractive index that is individually tuned to a specific desired value. A novel subwavelength high index contrast grating (HCG) as a surface-normal broadband reflector was later proposed by Chang-Hasnain $[23,24]$. An HCG has been shown to replace conventional DBRs for VCSELs and offers desirable attributes, including lithographically defined polarization [25] and wavelength [26], a large aperture while maintaining a single transverse mode $[25,27]$, and a large fabrication tolerance [28].

Here, we summarize recent approaches to achieve wide spectral bandwidth using a coupled microcavity, asymmetric tandem quantum wells, and high index contrast distributed Bragg-reflector and assess the trends in this field. We first address two light absorption optoelectronic devices, RCEPDs and EAMs, using a coupled cavity structure for wide response and transmittance, respectively. Next, absorption coupling using quantum wells of different thickness, called asymmetric tandem quantum wells, in an EAM for wide transmittance change is described. Finally, wide spectral bandwidth using oblique-angle deposition and high index contrast grating techniques is discussed.

\section{Flat Top Response of RCEPD}

WDM is a key technology for increasing the transmission capacity of optical fiber communication systems [29, 30]. A high-speed and high-gain demultiplexing receiver is a crucial part of these systems. A monolithic multiple wavelength demultiplexing device with a narrow interchannel wavelength spacing and low crosstalk between channels is essential for effective use of the available bandwidth.

Since 1991, the RCEPD, which is constructed by inserting an absorption layer into an integrated Fabry-Perot resonant cavity, has been established as a promising device for WDM applications because of its wavelength selectivity as well as its high-speed response. In particular, monolithic integration of VCSEL and RCEPD can reduce alignment time, lower packaging cost, and provide design flexibility (Figure 1(a)) $[31,32]$. The problem of misalignment of the cavity-mode wavelength of the emitter and RCEPD, however, significantly affects the system performance [30, 33-35]. As shown in Figure 1(b), misaligned operating wavelengths between the VCSEL and RCEPD can be changed by etching the top most layer of RCEPD $[33,36]$. However, the conventional RCEPD spectral response shapes are triangular, which affects the sensitivity of system performance because of the extremely narrow spectra of the VCSELs (Figure 1(c)). As shown in Figure $1(\mathrm{~d})$, there is also a tradeoff between the quantum efficiency and the interchannel crosstalk parameter in the conventional RCEPD [37]. To solve this issue, low loss 


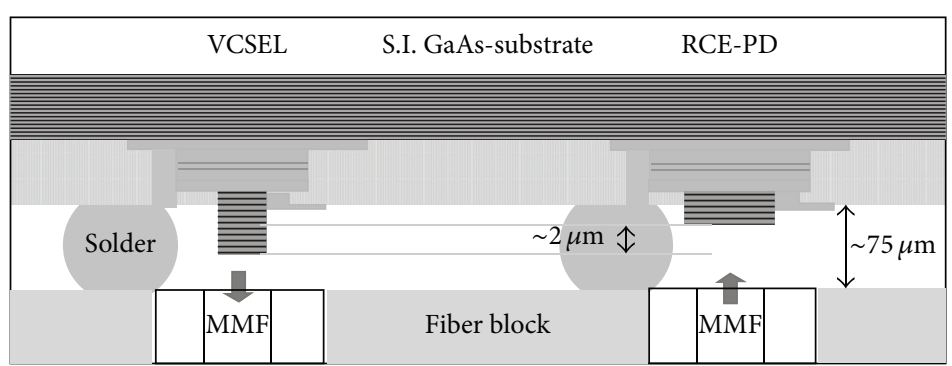

(a)

(c)

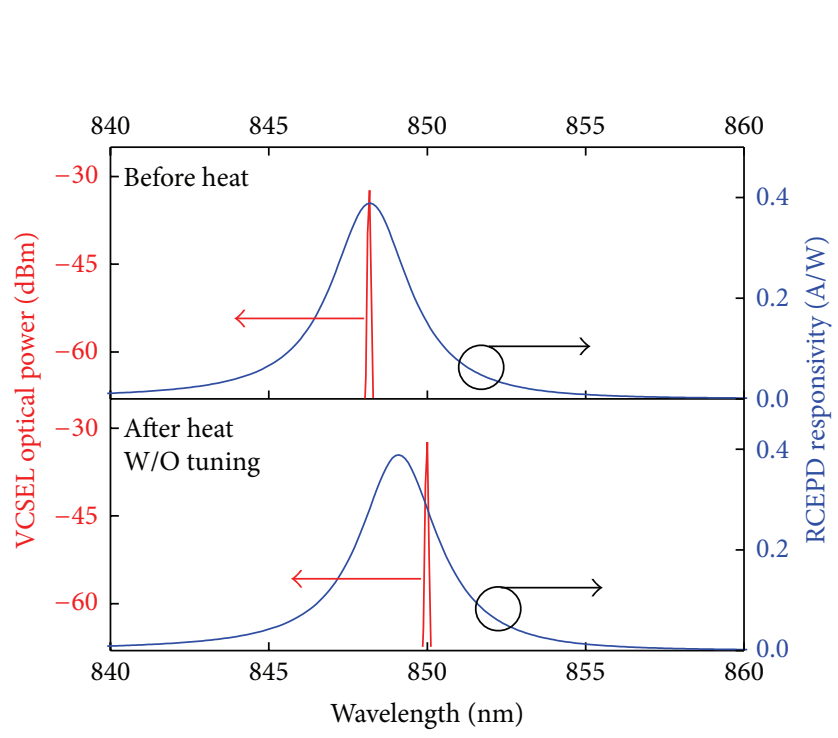

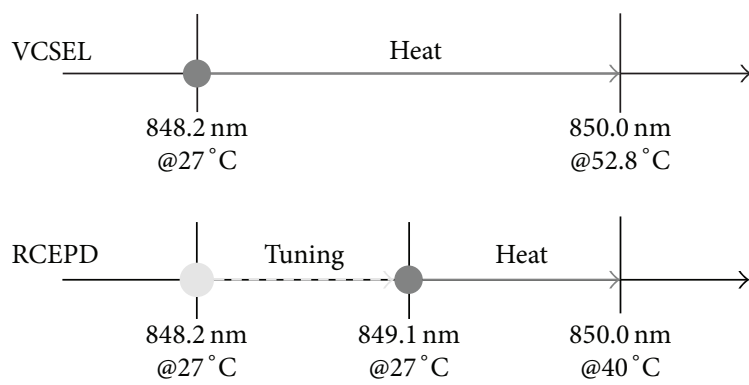

(b)

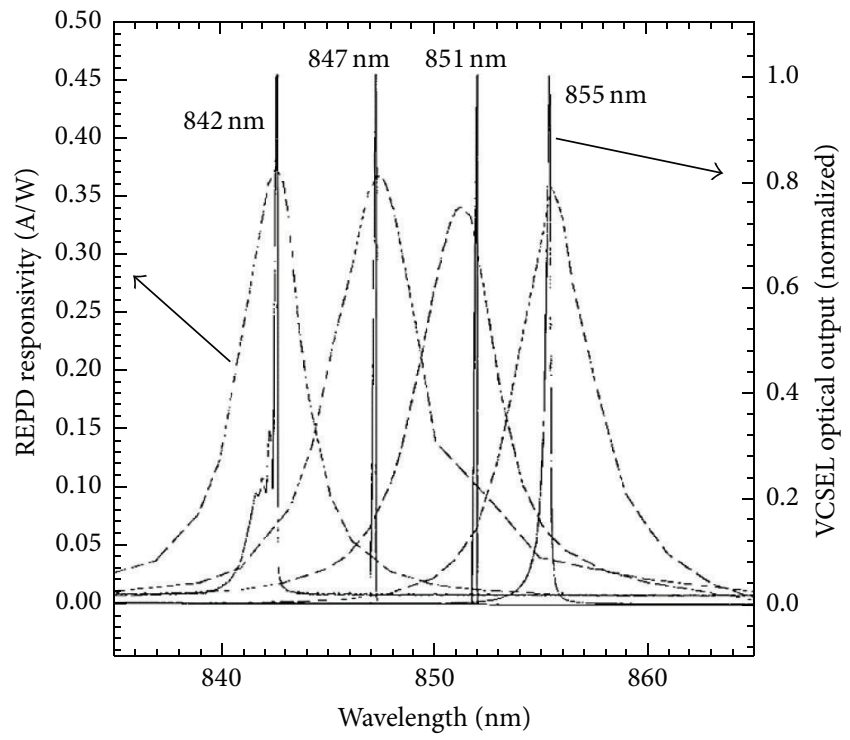

(d)

FIGURE 1: (a) The VCSEL array and RCEPD array are separately passive aligned with planar waveguides such as multimode fiber (MMF) [33]. (b) Schematic diagram of cavity-mode wavelength alignment by using cavity-mode tuning of RCEPD chips [33] and (c) the related spectra without alignment. (d) The measured responsivity and CW lasing spectra of four elements from two RCEPD and VCSEL arrays in close proximity to each other [34].

cavity is added to the top mirror and optically coupled in series with the absorptive cavity of the conventional single resonant cavity design $[10,11,38]$. This flat top response with sharp edges reduces the interchannel crosstalk and improves the accuracy and stability of the system, although lasing wavelength is changed.

When two or more Fabry-Perot cavities are placed in series, a double-peak transmittance curve can be obtained, which has a more promising response shape. This multiple half-wave cavities structures with three mirrors can be made of quarter-wave stacks of semiconductor compounds or dielectric materials. A theoretical analysis of the quantum efficiency of an InGaAs/GaAs RCEPD was presented by using this microcavity in place of the top DBR (Figure 2). The quantum efficiency can amount to $100 \%$ with a sufficiently large quantity of bottom mirror pairs in a conventional RCEPD (gray curve). On the other hand, the flat-topped quantum efficiency spectrum has been obtained in a coupled cavity RCEPD (black curve). This response can be explained in consideration of phase conditions in the resonator. The electromagnetic field between the mirrors almost does not depend on wavelength when the slope of the phase change is zero on the wavelength. When the thickness of coupled cavity is thicker than quarter-wave resonant thickness, the phase change for the wavelength is zero. As a result, the reflectivity shows the flatness near the resonance wavelength [13].

The location of the absorption layer affects the response curve. Two cavities have the same optical lengths, as integral times of half resonant wavelength. When the absorption layer is on the coupled cavity, the quantum efficiency curve is hollow and much deeper for the top-illuminated device (Figure 3). Because the light from the top of the device is significantly absorbed, there is no coupling effect of electric fields by the coupled cavity. Therefore, the coupled cavity is placed above the absorption cavity for a flat-topped response.

\section{Wide Spectral Bandwidth Electroabsorption Modulator}

Surface-normal EAMs based on the quantum confined Stark effect are prime candidates for applications such as threedimensional (3D) imaging systems [16, 39]. The development 

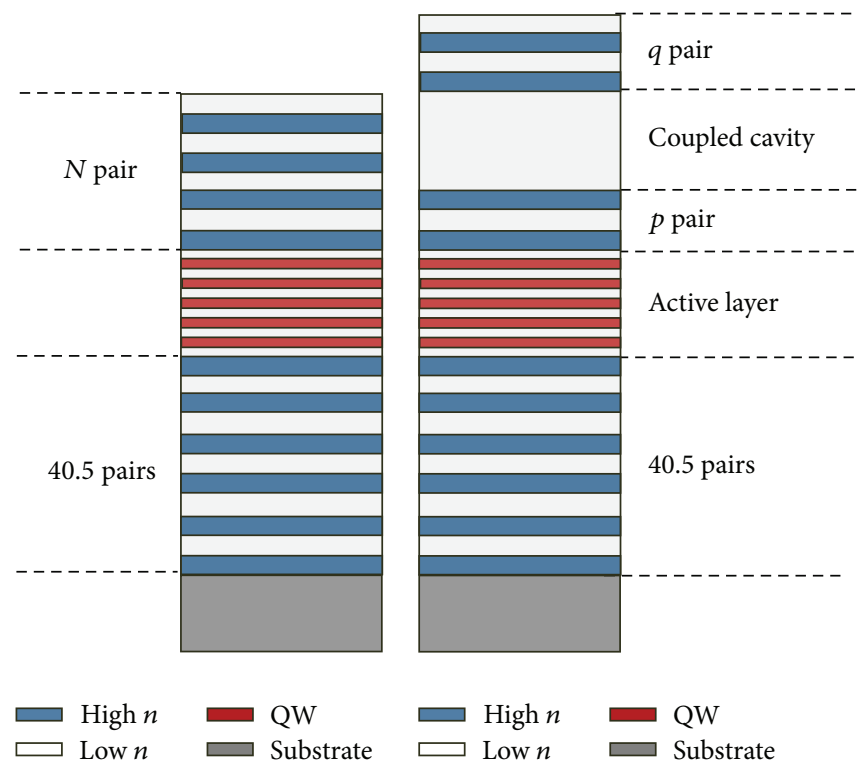

(b)

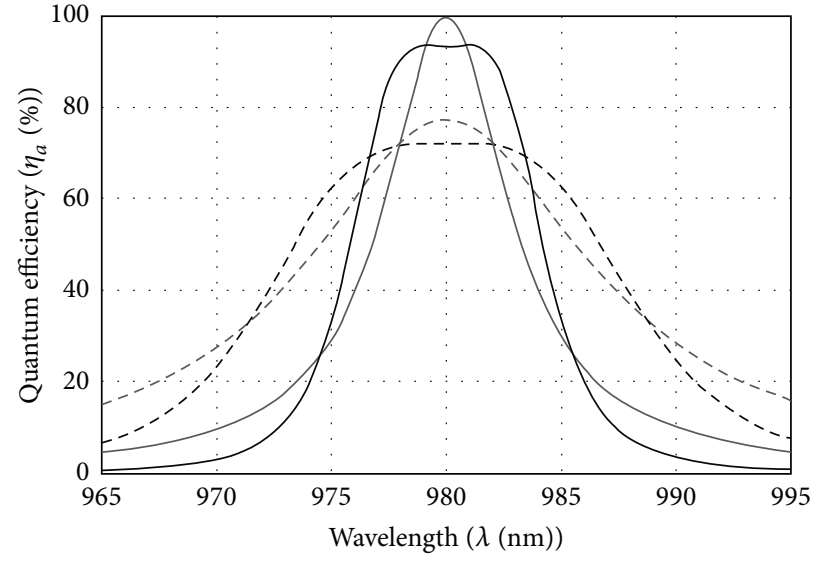

(c)

FIGURE 2: (a) The RCEPD structure with conventional RCEPD, (b) RCEPD with coupled cavity, and (c) calculated quantum efficiency of two RCEPDs. Grey curves illustrate results of calculation of conventional RCEPDs, where $N=8$ (solid curve) and $N=3$ (dashed curve). Black curves illustrate results of calculation of RCEPD with coupled cavity, where $p=21, q=4.5$ (solid curve) and $p=15, q=1.5$ (dashed curve) [13].

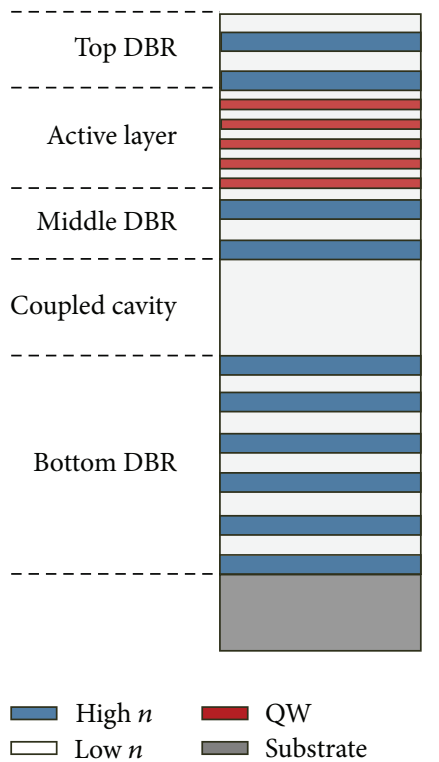

(a)

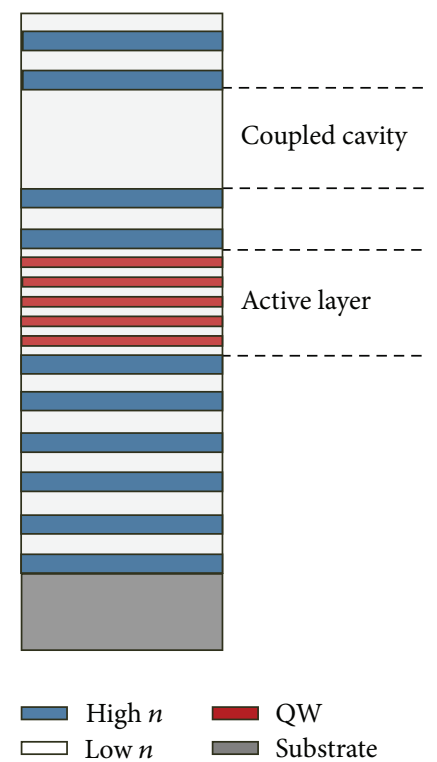

(b)

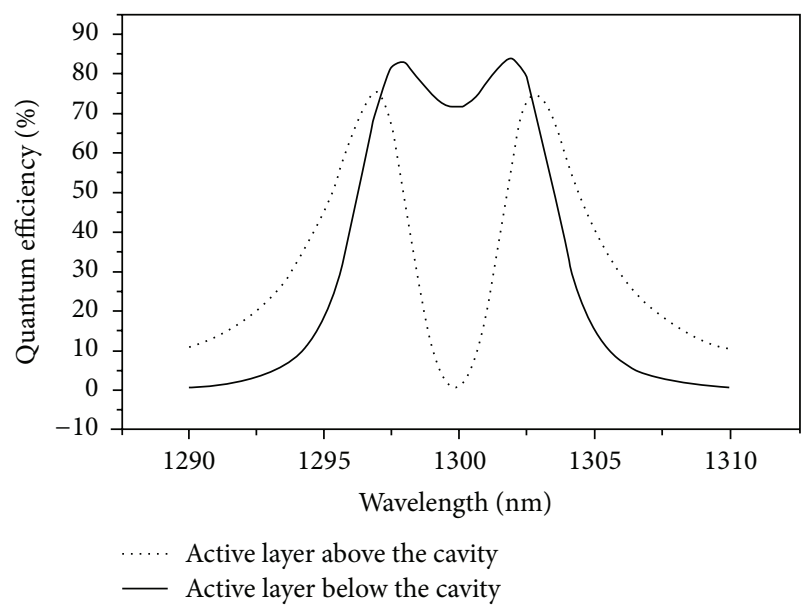

(c)

FIGURE 3: (a) The RCEPD structure with the active layer above the cavity, (b) RCEPD structure with the active layer below the cavity, and (c) the calculated quantum efficiency of the two structures [12].

of an optical shutter using an EAM to obtain high resolution depth information in a time of flight based 3D imaging system was recently reported as shown in Figure $4[16,40]$. Despite improving the modulation speed and depth, EAMs still have relatively narrow spectral bandwidth produced by the Fabry-Perot cavity. A narrow spectral bandwidth reduces the manufacturing tolerance of the $3 \mathrm{D}$ imaging system and the operating temperature range, because the bandgap and refractive index of a semiconductor material change as a function of the temperature. Thus, the EAM should be able to operate over a wide spectral bandwidth with high modulation depth in order to improve the reliability of $3 \mathrm{D}$ imaging systems. A thick absorption region results in wide spectral bandwidth of the EAM, but this results in a high 


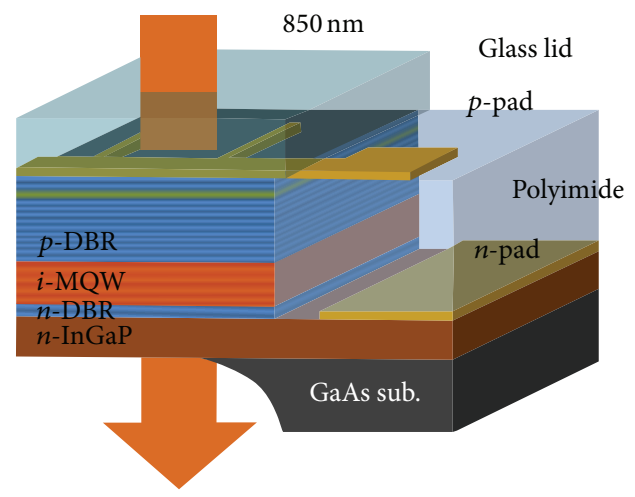

(a)

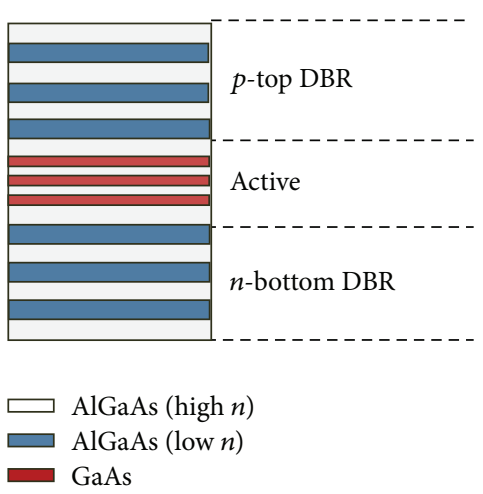

(b)

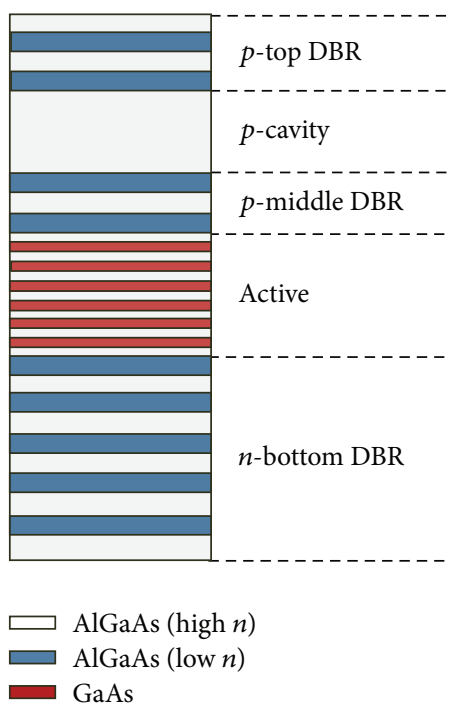

(c)

FIGURE 4: (a) Top view image and cross-sectional schematic of $850 \mathrm{~nm}$ EAM [40] and (b) designed single-cavity and (c) coupled microcavity EAM structures.

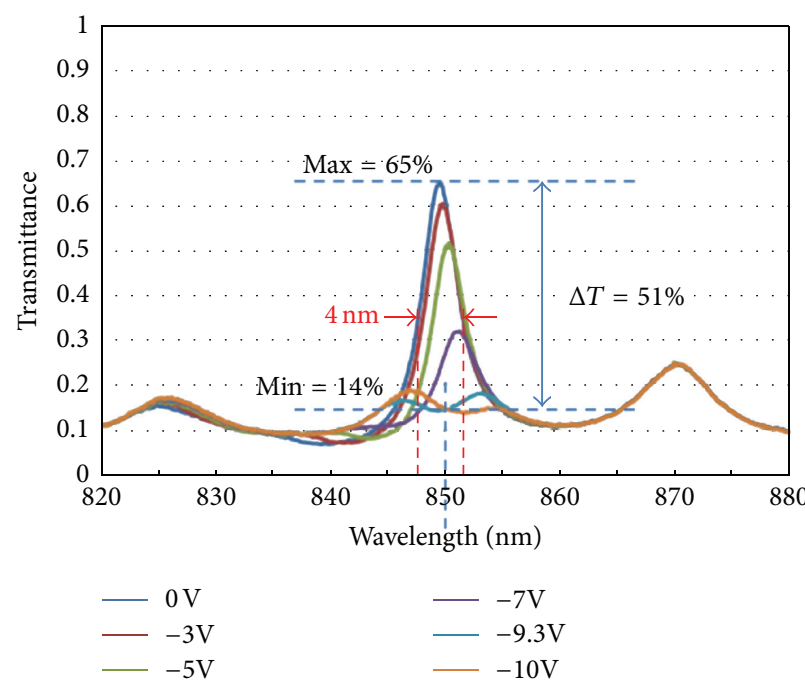

(a)
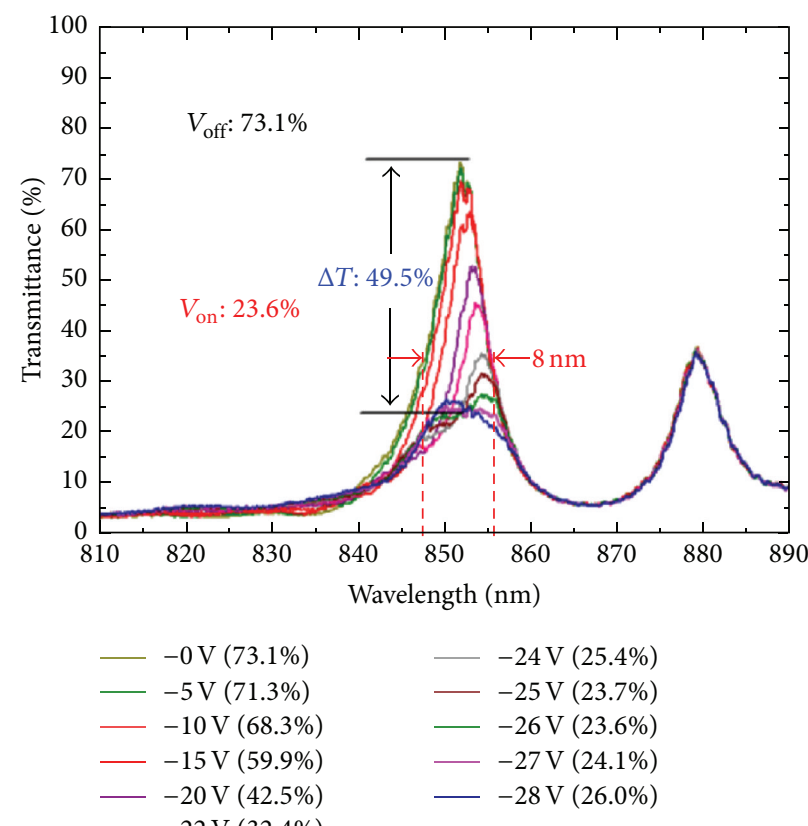

(b)

FIGURE 5: (a) Transmittance measurement for characterization of transmittance variation of the EAM for single-cavity structure [16] and (b) coupled microcavity structure [40].

operating voltage. To solve this issue, a coupled Fabry-Perot cavity structure used in band-pass filters [41-43] allows the realization of a rectangular transmittance band, leading to a broad and flat spectrum for high transmittance. And the measured transmittances of the EAM for single-cavity and coupled microcavity structures are shown in Figures 5(a) and 5(b), respectively. Under zero bias, the spectral bandwidth of the coupled microcavity is about $8 \mathrm{~nm}$, which is two times wider than that of the single-cavity structure. Furthermore, the transmittance change for the coupled microcavity EAM is comparable with that of the single-cavity structure. However to achieve $50 \%$ transmittance change in coupled microcavity 

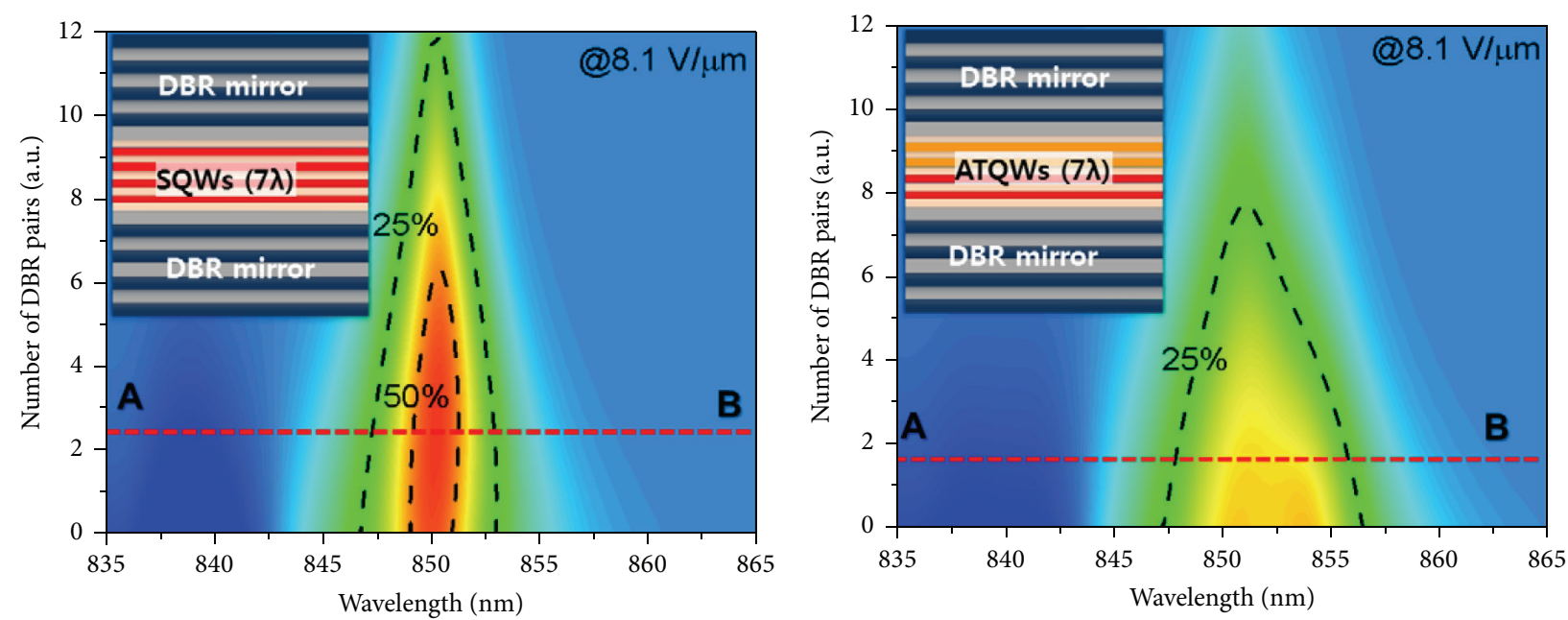

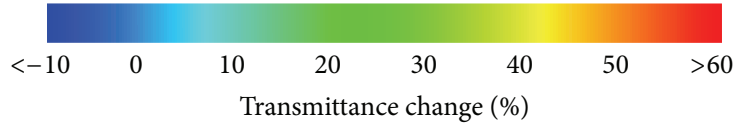

(a)

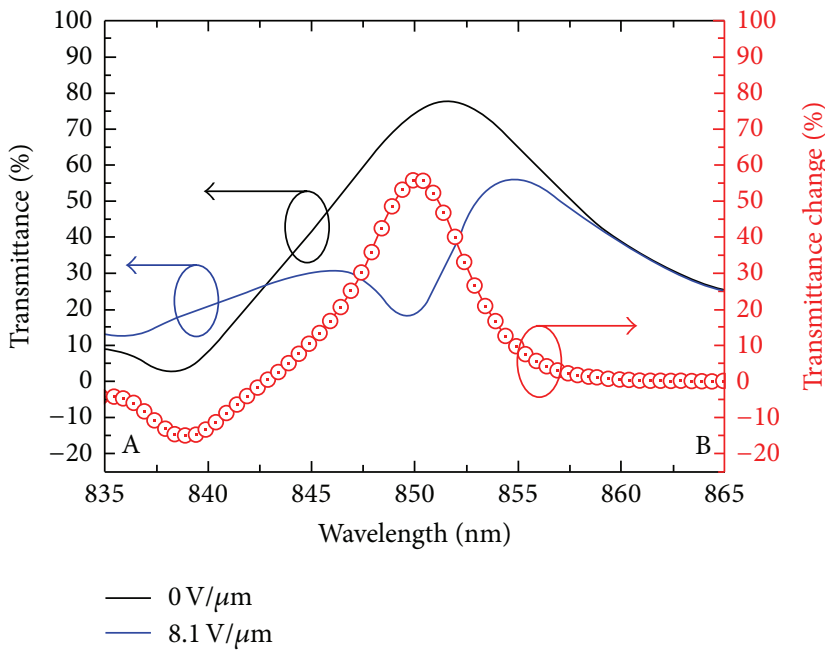

(c)

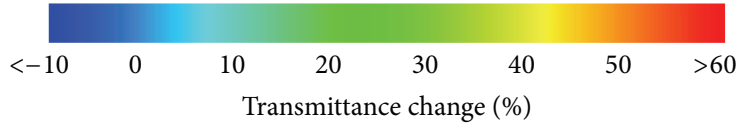

(b)

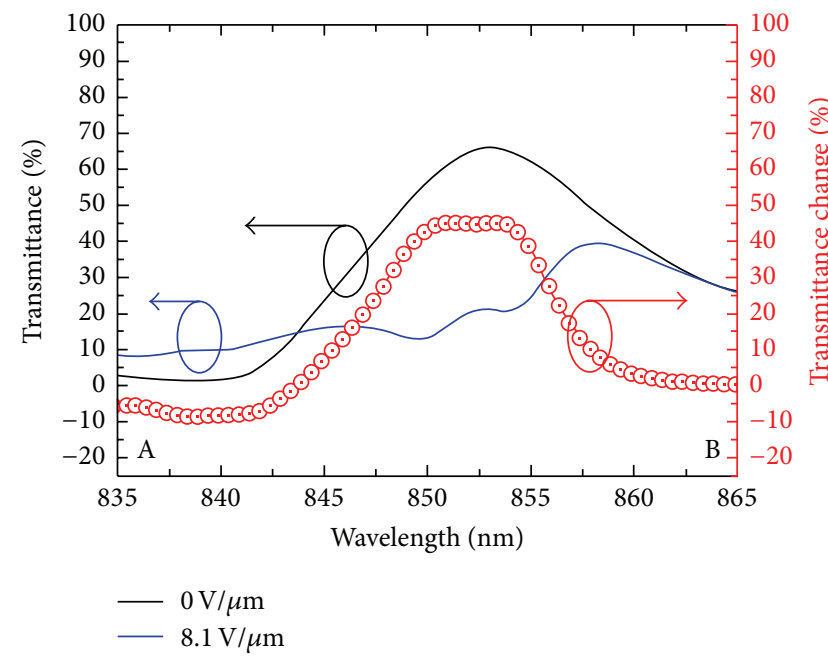

(d)

FIGURE 6: Contour plots of the calculated transmittance change as a function of the number of pairs of DBR for a 7- $\lambda$ SC structure with (a) $8 \mathrm{~nm}$ thick SQWs and (b) $8 / 8.5 \mathrm{~nm}$ ATQWs. The transmittance and transmittance change at the dashed line AB of the (c) $8 \mathrm{~nm}$ thick SQWs and the (d) $8 / 8.5 \mathrm{~nm}$ ATQWs. The insets of (a) and (b) schematically illustrate a SC structure with $8 \mathrm{~nm}$ thick SQWs and 8/8.5 nm ATQWs, respectively [61].

structure, the pairs of QWs are increased, which result in high operating voltage. There is a tradeoff between transmittance contrast and operating voltage in EAM structure.

Another approach to broaden the spectral bandwidth is to use asymmetric tandem quantum wells (ATQWs). Whereas the above techniques use coupling of the electric field in a DBR structure under zero bias, ATQW s use coupling of light absorption in the active region under reverse bias. Figure 6 shows the contour plots and transmittance changes as a function of DBR pairs for a $7-\lambda$ single-cavity (SC) structure of $8 \mathrm{~nm}$ thick single quantum wells (SQWs) and $8 / 8.5 \mathrm{~nm}$ thick ATQWs. The transmittance of the EAM decreases under reverse bias because of absorption of the quantum well. As shown in Figure 6(d), there are two absorption resonances transmittance peaks under bias with ATQWs. It is noteworthy that the quantum wells with different thickness absorb broad wavelength. As a result, the spectral bandwidth of the transmittance change for a single cavity with ATQWs was improved in comparison with the single-cavity structure with SQWs.

\section{Wide Stop Band of DBR}

Normally DBRs consist of low index layers alternating with high index layers. The semiconductor DBRs are grown by molecular beam epitaxy or metal organic chemical vapor 

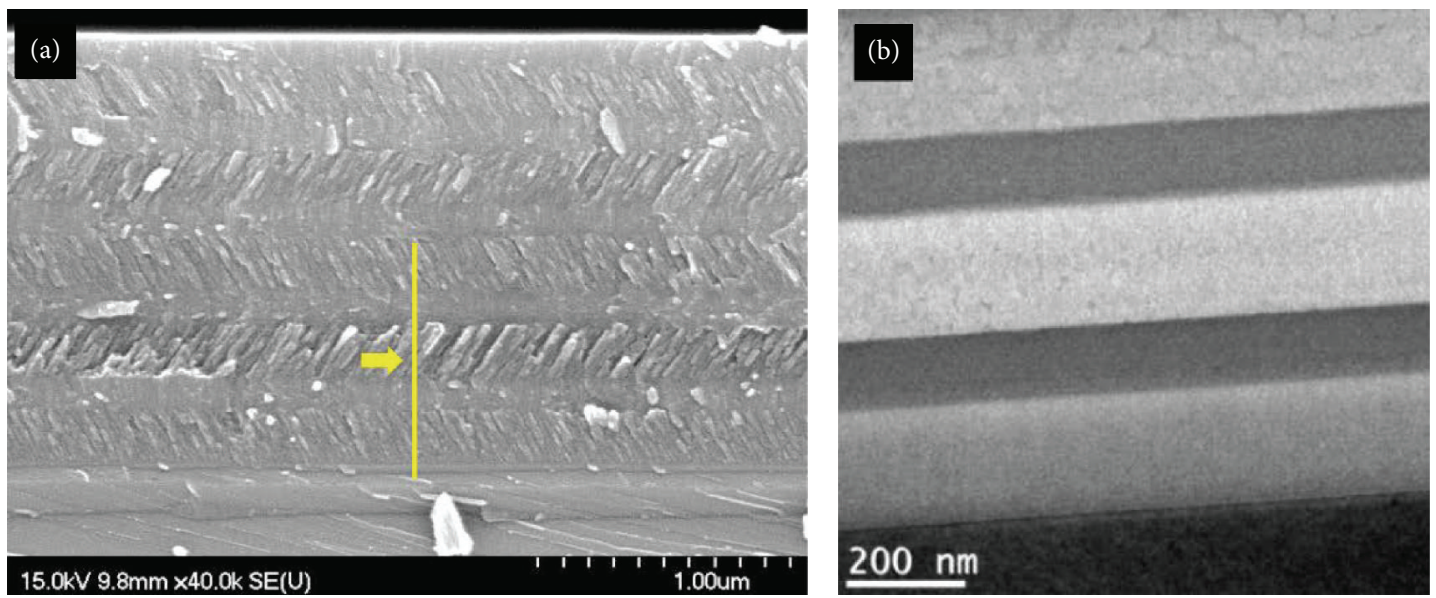

FIGURE 7: (a) SEM image of 5-period a-Si/a-Si DBR fabricated on Si substrate. The yellow line is an enlarged cross-sectional TEM image, and an image of an a-Si DBR is shown in (b) [46].

deposition equipment for single crystal quality. For high reflectivity, semiconductor DBR pairs must be repeated many times, in excess of 20 pairs, because of the low index contrast of the semiconductor DBR. Thickness control is thus an important parameter because it can change the spectral stop band and reflectivity. To address this, dielectric materials with high index contrast are used for a wide stop band of reflectivity. These dielectric DBRs demonstrate a wide stop band with high reflectivity using relatively few layers.

Figure 7 shows a-Si layers deposited on a Si substrate by an e-beam evaporator using inclined sample holders for tilted angle evaporation. The refractive index of the deposited film decreases as the oblique angle increases in the OAD process $[44,45]$. First, a low index layer was deposited with a tilted angle. The nanocolumnar layers have a separate pattern, as shown in a cross-sectional scanning electron microscope (SEM) image. The interface between the low and high index layers is relatively good in terms of distinguishing the interface, as seen in the TEM image; hence, the refractive index of the layers was decreased in a controllable manner.

For comparison, the reflectivity values of DBRs with other general material systems were calculated, and the results are shown in Figure 8(a). The spectral bandwidth and reflectivity of a dielectric $\mathrm{SiO}_{2} / \mathrm{TiO}_{2} \mathrm{DBR}(\Delta n=1.0)$ are broader and higher than those of a semiconductor GaAs/AlAs DBR $(\Delta n=$ $0.48)$. In an a-Si/a-Si DBR $(\Delta n=1.65)$ structure, almost $100 \%$ reflectivity was observed in a wavelength range from $1400 \mathrm{~nm}$ to $1800 \mathrm{~nm}$, and good agreement between the calculated and measured results was observed. Figure $8(\mathrm{~b})$ shows the reflectivity spectra of fabricated a-Si/a-Si DBRs as a function of the number of periods. A three-period a-Si/a-Si DBR was sufficient to ensure high reflectivity that exceeded $97 \%$.

The reflective property of the $2^{\prime \prime}$ full-wafer a-Si/a-Si DBR with five periods was investigated experimentally [46]. The relative reflectivity mapping was measured at a center wavelength of $1550 \mathrm{~nm}$ (Figure 9(a)). There is only a small variation in the measured relative reflectivity with relatively uniform reflectivity over the full-wafer. The measured reflectivity of each position is marked in Figure 9(b) by spectrophotometry measurement. There was little difference in the measured reflectivity of each position. A wide stop band $(800 \mathrm{~nm})$ with only a five-period a-Si/a-Si DBR was achieved experimentally. Therefore, a-Si/a-Si DBR is practical and innovative for cost-effective optical components capable of high levels of reflection.

Recent innovations of one-dimensional and high-contrast gratings (HCG) as a surface-normal broadband reflector were reported. This grating provides a high contrast in the refractive indices for the grating medium and its surrounding materials. This HCG can replace conventional DBRs for VCSELs, as shown in Figure 10 [26, 28, 45, 47-52], tunable VCSELs [47, 53-55], and high-Q optical resonators [56]. Also, by removing the quantum wells and turning the device such that it is totally passive, all-pass filters can be constructed as phase tuners [57]. These tunable devices can be further fabricated via large scale photonic integration. By replacing the DBR with HCG, the manufacturing cost can be reduced due to thinner epitaxial layers and the microelectromechanical structure (MEMS) footprint also can be reduced, leading to a more compact structure and higher integration capability.

The design parameters for the structure include the indices of refraction of the high and low index materials, grating period, thickness, and duty cycle [25, 58-60]. When the period of the grating becomes smaller than the incident wavelength, which is known as a subwavelength grating, only the zeroth diffraction order exists in the reflected and transmitted waves and all the higher diffraction orders are evanescent modes [58]. This subwavelength grating will basically operate as a simple mirror. When the reflective index of grating material has high contrast to surroundings, the high and broad reflectivity are possible [59]. The broad reflectivity can be optimized for one E-field orientation as shown in Figure 11. This characteristic of polarization control can be designed with simple HCG structure. 


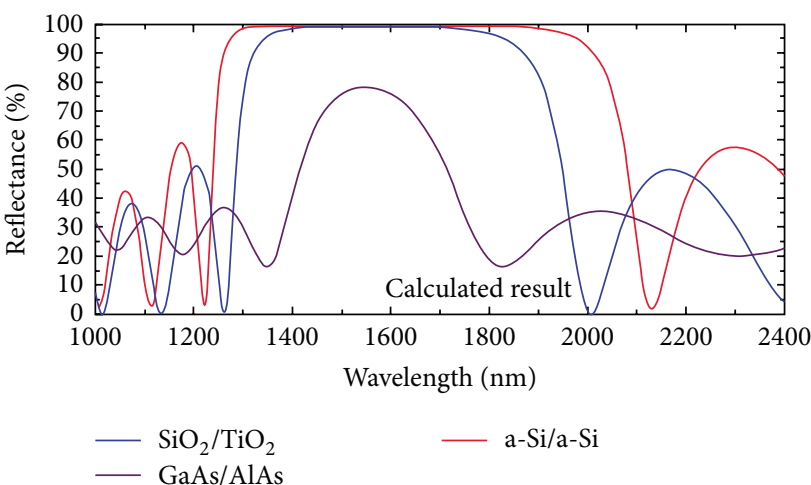

(a)

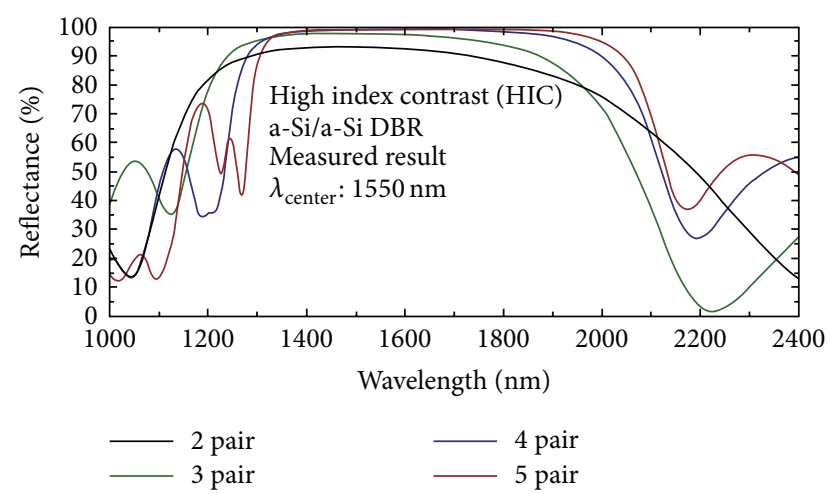

(b)

Figure 8: (a) Calculated reflectivity spectra of GaAs/AlAs, $\mathrm{SiO}_{2} / \mathrm{TiO}_{2}$, and a-Si/a-Si DBRs as a function of wavelength. (b) Measured reflectivity spectra of 2-, 3-, 4-, and 5-period a-Si/a-Si DBRs as a function of wavelength. The center wavelength of every DBR was $1550 \mathrm{~nm}$ [46].
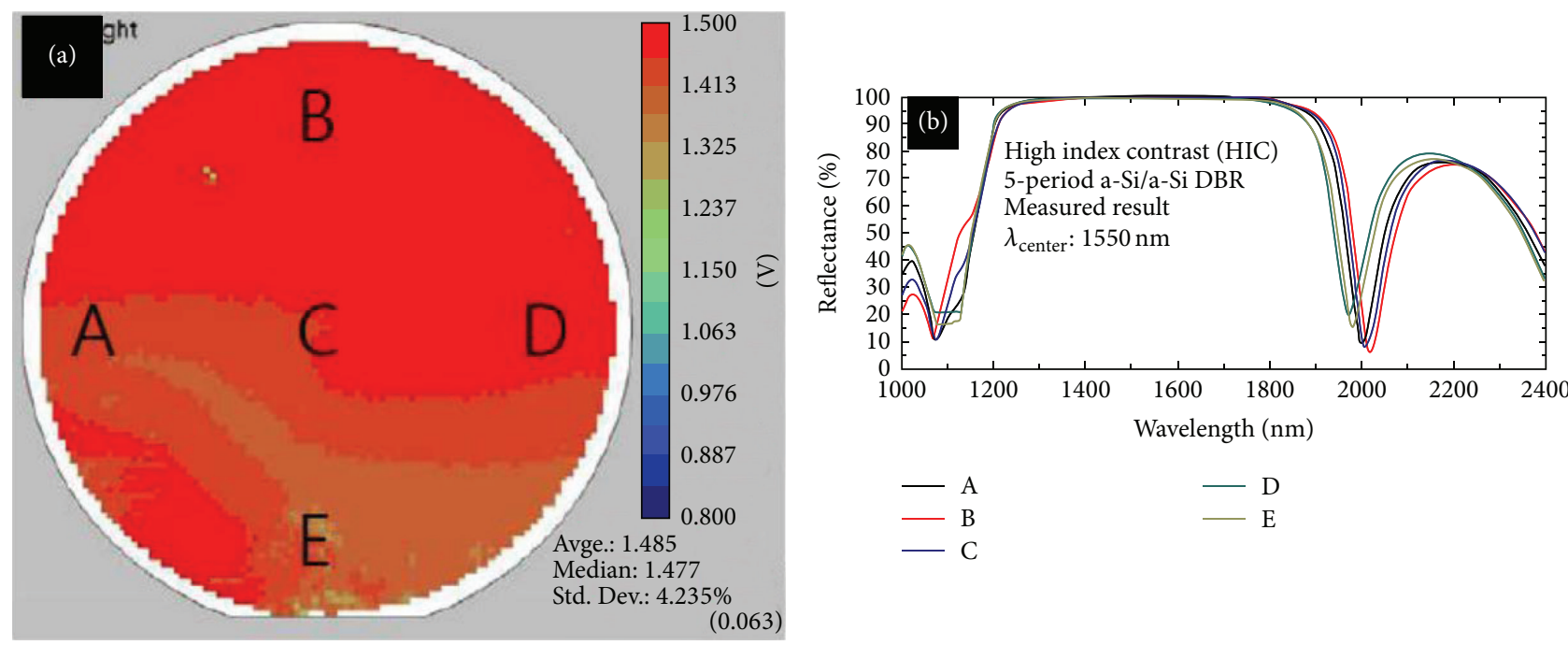

FIGURE 9: (a) The relative reflectance mapping image of a $2^{\prime \prime}$ full-wafer DBR fabricated on a Si substrate. The letters A-E represent each position for measuring the reflectivity. (b) The measured reflectivity of all positions of the DBR fabricated on a $2^{\prime \prime} \mathrm{Si}$ wafer as a function of wavelength [46].

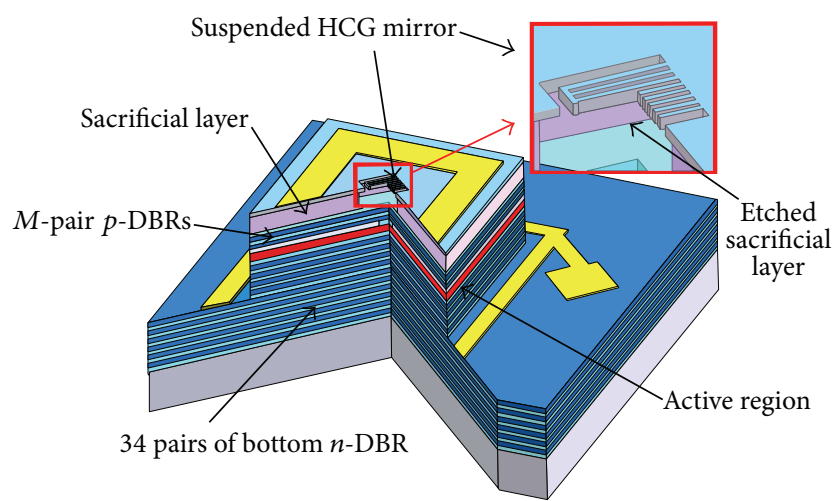

FIGURE 10: Schematic of a typical $850 \mathrm{~nm}$ design, consisting of an HCG-based top mirror, a $5 / 4-\lambda$ air gap, a $\lambda$-cavity containing an active region, and a conventional semiconductor-based bottom $n$ DBR mirror. Here $M=2$ or 4 [47].

\section{Summary}

Recently developed techniques for wide spectral bandwidth that make it possible to realize multiple functionalities are reviewed. The flat-topped response of a RCEPD with a coupled cavity can reduce the channel crosstalk and is not sensitive to wavelength fluctuation in the transmitter or resonant control in the receiver in a WDM system. Meanwhile, an EAM with a coupled cavity as an optical shutter shows a wide transmittance spectrum without a bias state. Moreover, ATQWs that consist of quantum wells with different thickness coupling light absorption under reverse bias result in a wide transmittance change. These two structures help the EAM in 3D imaging systems which require operation with high reliability over a wide temperature range. To increase the reflectivity over a wide wavelength range, the use of high index contrast materials in tandem with oblique-angle 


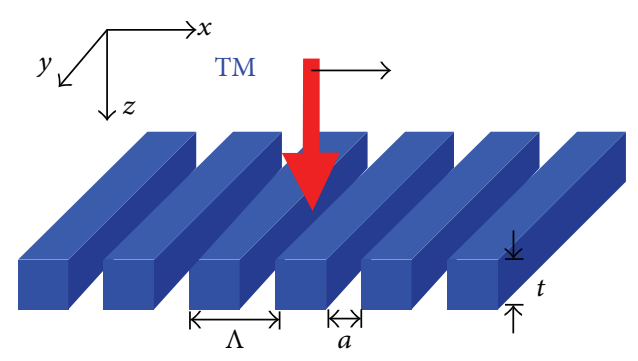

(a)

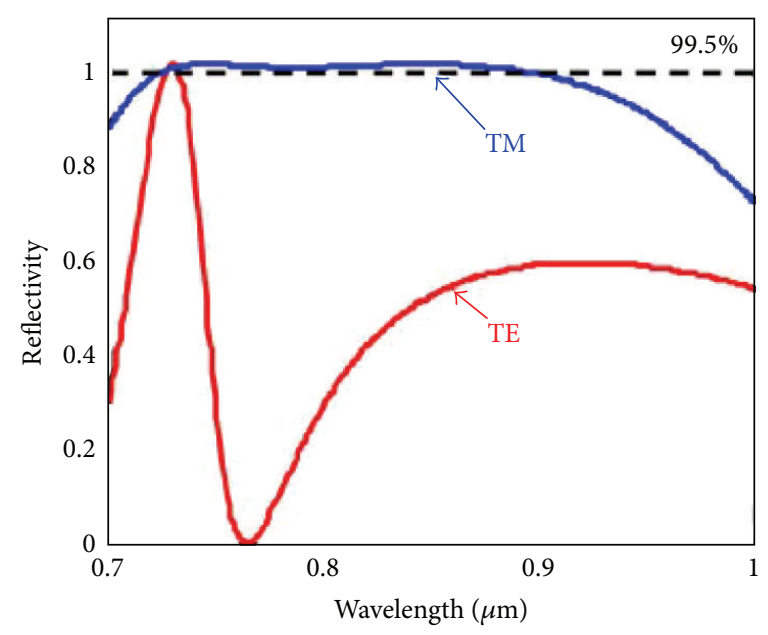

(c)

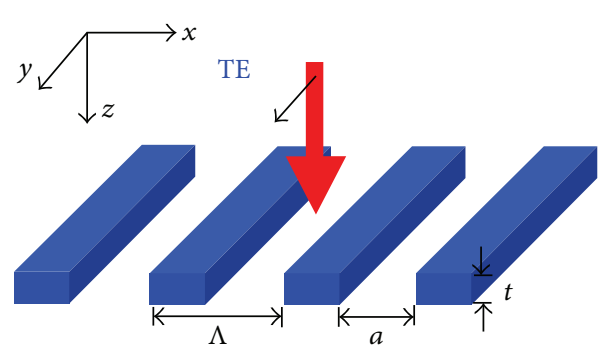

(b)

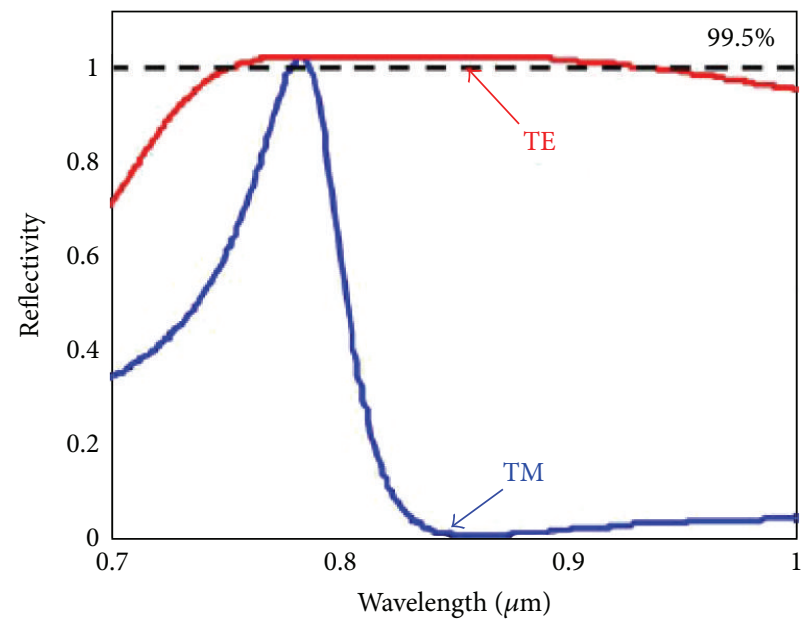

(d)

Figure 11: Schematic of (a) TM-polarized $(\Lambda=380 \mathrm{~nm}, a=130 \mathrm{~nm}$, and $t=235 \mathrm{~nm})$ and (b) TE-polarized $(\Lambda=620 \mathrm{~nm}, a=400 \mathrm{~nm}$, and $t=140 \mathrm{~nm}$ ) HCG. Red arrows show the light propagation directions. The black arrows show the E-field polarization directions. Calculated reflectivity spectra for surface-normal incident plane waves with E-field along the $x$ (blue) and $y$ (red) directions for (c) TM- and (d) TEHCG. The TM-HCG has a very high reflectivity for E-field aligned in the $x$ direction but significantly lower reflectivity for the $y$ direction. The opposite is true for TE-HCG. This enables polarization selection in HCG-VCSELs [25].

deposition, which is independent of size, features, and substrate, will provide an interesting new method that may achieve improved performance in practical applications such as light emitting diodes, VCSELs, and photovoltaics. Also, by replacing the DBR with HCG, manufacturing costs can be reduced due to thinner epitaxial layers and the MEMS footprint can be reduced, leading to a more compact structure and higher integration capability. Furthermore an HCG could be a key component for producing devices that require particularly pure polarization characteristics. The reviewed approaches and design guidelines for wide spectral bandwidth in resonant cavity optoelectronic devices can be readily merged with a variety of other optoelectronic devices.

\section{Conflict of Interests}

The authors declare that there is no conflict of interests regarding the publication of this paper.

\section{Acknowledgments}

This work was partly supported by the Center for Integrated Smart Sensors funded by the Ministry of Science,
ICT \& Future Planning as Global Frontier Project (CISS2013M3A6A6073718) and by Basic Science Research Program through the National Research Foundation of Korea (NRF) funded by the Ministry of Science, ICT \& Future Planning (2014R1A1A1005945) and by BK21PLUS, Creative Human Resource Development Program for IT Convergence.

\section{References}

[1] T. Jeong, H. H. Lee, S.-H. Park, J. H. Baek, and J. K. Lee, "InGaN/AlGaN ultraviolet light-emitting diode with a $\mathrm{Ti}_{3} \mathrm{O}_{5} / \mathrm{Al}_{2} \mathrm{O}_{3}$ distributed Bragg reflector," Japanese Journal of Applied Physics, vol. 47, no. 12, pp. 8811-8814, 2008.

[2] B. Temelkuran, E. Ozbay, J. P. Kavanaugh, G. Tuttle, and K. M. Ho, "Resonant cavity enhanced detectors embedded in photonic crystals," Applied Physics Letters, vol. 72, no. 19, pp. 2376-2378, 1998.

[3] M. Lončar, T. Yoshie, A. Scherer, P. Gogna, and Y. Qiu, "Lowthreshold photonic crystal laser," Applied Physics Letters, vol. 81, no. 15 , pp. 2680-2682, 2002.

[4] Y. M. Song, K. S. Chang, B. H. Na, J. S. Yu, and Y. T. Lee, "Low thermal resistance, high-speed $980 \mathrm{~nm}$ asymmetric intracavitycontacted oxide-aperture VCSELs," Physica Status Solidi (A), vol. 206, no. 7, pp. 1631-1635, 2009. 
[5] P. R. Villeneuve, D. S. Abrams, S. Fan, and J. D. Joannopoulos, "Single-mode waveguide microcavity for fast optical switching," Optics Letters, vol. 21, no. 24, pp. 2017-2019, 1996.

[6] A. Sharkawy, S. Shi, and D. W. Prather, "Multichannel wavelength division multiplexing with photonic crystals," Applied Optics, vol. 40, no. 14, pp. 2247-2252, 2001.

[7] G. T. Paloczi, J. Scheuer, and A. Yariv, "Compact microringbased wavelength-selective inline optical reflector," IEEE Photonics Technology Letters, vol. 17, no. 2, pp. 390-392, 2005.

[8] S. T. Chu, B. E. Little, W. Pan, T. Kaneko, S. Sato, and Y. Kokubun, "An eight-channel add-drop filter using vertically coupled microring resonators over a cross grid," IEEE Photonics Technology Letters, vol. 11, no. 6, pp. 691-693, 1999.

[9] M. S. Ünlü and S. Strite, "Resonant cavity enhanced photonic devices," Journal of Applied Physics, vol. 78, no. 2, pp. 607-639, 1995.

[10] S. Y. Hu, E. R. Hegblom, and L. A. Coldren, "Coupledcavity resonant photodetectors for high-performance wavelength demultiplexing applications," Applied Physics Letters, vol. 71, no. 2, pp. 178-180, 1997.

[11] C.-H. Chen, K. Tetz, and Y. Fainman, "Resonant-cavityenhanced $\mathrm{p}$-i-n photodiode with a broad quantum-efficiency spectrum by use of an anomalous-dispersion mirror," Applied Optics, vol. 44, no. 29, pp. 6131-6140, 2005.

[12] Y. Zhong, Z. Pan, L. Li, Y. Huang, and X. Ren, "Proposition of a nearly rectangular response resonant cavity enhanced (RCE) photodetector," in Semiconductor Optoelectronic Device Manufacturing and Applications, vol. 4602 of Proceedings of SPIE, pp. 74-78, Nanjing, China, November 2001.

[13] S. V. Gryshchenko, A. A. Dyomin, and V. V. Lysak, “Theoretical study of the quantum efficiency of InGaAs/GaAs resonant cavity enhanced photodetectors," in Proceedings of the International Workshop on Optoelectronic Physics and Technology (OPT '07), pp. 20-22, IEEE, Kharkov, Ukraine, June 2007.

[14] R. Lange and P. Seitz, "Solid-state time-of-flight range camera," IEEE Journal of Quantum Electronics, vol. 37, no. 3, pp. 390-397, 2001.

[15] S. Gokturk, H. Yalcin, and C. Bamji, "A time-of-flight depth sensor-system description, issues and solutions," in Proceedings of the Conference on Computer Vision and Pattern Recognition Workshop, p. 35, Washington, DC, USA, June 2004.

[16] Y.-H. Park, Y.-C. Cho, J.-W. You et al., "Micro-optical system based 3D imaging for full HD depth image capturing," in MOEMS and Miniaturized Systems XI, vol. 8252 of Proceedings of SPIE, San Francisco, Calif, USA, January 2012.

[17] J. M. Dallesasse, N. Holonyak Jr., A. R. Sugg, T. A. Richard, and N. El-Zein, "Hydrolyzation oxidation of AlxGal-xAs-AlAsGaAs quantum well heterostructures and superlattices," Applied Physics Letters, vol. 57, no. 26, pp. 2844-2846, 1990.

[18] A. R. Sugg, N. Holonyak Jr., J. E. Baker, F. A. Kish, and J. M. Dallesasse, "Native oxide stabilization of AlAs-GaAs heterostructures," Applied Physics Letters, vol. 58, no. 11, pp. 1199-1201, 1991.

[19] G. W. Pickrell, J. H. Epple, K. L. Chang, K. C. Hsieh, and K. Y. Cheng, "Improvement of wet-oxidized $\mathrm{Al}_{x} \mathrm{Ga}_{1-x}$ As $(x \sim 1)$ through the use of AlAs/GaAs digital alloys," Applied Physics Letters, vol. 76, no. 18, pp. 2544-2546, 2000.

[20] M. J. Brett and M. M. Hawkeye, "Materials science: new materials at a glance," Science, vol. 319, no. 5867, pp. 1192-1193, 2008.
[21] J.-Q. Xi, M. F. Schubert, J. K. Kim et al., "Optical thin-film materials with low refractive index for broadband elimination of Fresnel reflection," Nature Photonics, vol. 1, no. 3, pp. 176-179, 2007.

[22] J. K. Kim, T. Gessmann, E. F. Schubert et al., "GaInN lightemitting diode with conductive omnidirectional reflector having a low-refractive-index indium-tin oxide layer," Applied Physics Letters, vol. 88, no. 1, Article ID 013501, 2006.

[23] C. F. R. Mateus, M. C. Y. Huang, Y. Deng, A. R. Neureuther, and C. J. Chang-Hasnain, "Ultrabroadband mirror using low-index cladded subwavelength grating," IEEE Photonics Technology Letters, vol. 16, no. 2, pp. 518-520, 2004.

[24] C. F. R. Mateus, M. C. Y. Huang, L. Chen, C. J. Chang-Hasnain, and Y. Suzuki, "Broad-band mirror (1.12-1.62 $\mu \mathrm{m})$ using a subwavelength grating," IEEE Photonics Technology Letters, vol. 16, no. 7, pp. 1676-1678, 2004.

[25] C. J. Chang-Hasnain, Y. Zhou, M. C. Y. Huang, and C. Chase, "High-contrast grating VCSELs," IEEE Journal on Selected Topics in Quantum Electronics, vol. 15, no. 3, pp. 869-878, 2009.

[26] V. Karagodsky, B. Pesala, C. Chase, W. Hofmann, F. Koyama, and C. J. Chang-Hasnain, "Monolithically integrated multiwavelength VCSEL arrays using high-contrast gratings," Optics Express, vol. 18, no. 2, pp. 694-699, 2010.

[27] C. Chase, Y. Rao, W. Hofmann, and C. J. Chang-Hasnain, "1550 nm high contrast grating VCSEL," Optics Express, vol. 18, no. 15, pp. 15461-15466, 2010.

[28] Y. Zhou, M. C. Y. Huang, and C. J. Chang-Hasnain, "Large fabrication tolerance for VCSELs using high-contrast grating," IEEE Photonics Technology Letters, vol. 20, no. 6, pp. 434-436, 2008.

[29] C. A. Brackett, "Dense wavelength division multiplexing networks: Principles and applications," IEEE Journal on Selected Areas in Communications, vol. 8, no. 6, pp. 948-964, 1990.

[30] A. Alduino, Y. Zhou, S. Luong, C. P. Hains, and J. Cheng, "Wavelength multiplexing and demultiplexing using multiwavelength VCSEL and resonance-enhanced photodetector arrays," IEEE Photonics Technology Letters, vol. 10, no. 9, pp. 1310-1312, 1998.

[31] Y. M. Song, B. K. Jeong, B. H. Na, K. S. Chang, J. S. Yu, and Y. T. Lee, "High-speed characteristics of vertical cavity surface emitting lasers and resonant-cavity-enhanced photodetectors based on intracavity-contacted structure," Applied Optics, vol. 48, no. 25, pp. F11-F17, 2009.

[32] B. K. Jeong, Y. M. Song, V. V. Lysak, and Y. T. Lee, "Large area InGaAs/GaAs resonant cavity enhanced photodetector for sensor application," Journal of Optoelectronics and Advanced Materials, vol. 10, no. 10, pp. 2547-2554, 2008.

[33] I.-S. Chung and Y. T. Lee, "A method to tune the cavity-mode wavelength of resonant cavity-enhanced photodetectors for bidirectional optical interconnects," IEEE Photonics Technology Letters, vol. 18, no. 1, pp. 46-48, 2006.

[34] S. O. Luong, G. G. Ortiz, Y. Zhou et al., "Monolithic wavelengthgraded VCSEL and resonance-enhanced photodetector arrays for parallel optical interconnects," IEEE Photonics Technology Letters, vol. 10, no. 5, pp. 642-644, 1998.

[35] Y. Zhou, J. Cheng, and A. A. Allerman, "High-speed wavelength-division multiplexing and demultiplexing using monolithic quasi-planar VCSEL and resonant photodetector arrays with strained InGaAs quantum wells," IEEE Photonics Technology Letters, vol. 12, no. 2, pp. 122-124, 2000.

[36] Y. M. Song, K. S. Chang, B. H. Na, J. S. Yu, and Y. T. Lee, "Precise etch-depth control of microlens-integrated intracavity 
contacted vertical-cavity surface-emitting lasers by in-situ laser reflectometry and reflectivity modeling," Thin Solid Films, vol. 517, no. 19, pp. 5773-5778, 2009.

[37] K. Kishino, M. S. Unlu, J.-I. Chyi, J. Reed, L. Arsenault, and H. Morkoc, "Resonant cavity-enhanced (RCE) photodetectors," IEEE Journal of Quantum Electronics, vol. 27, no. 8, pp. 20252034, 1991.

[38] M. Gokkavas, G. Ulu, O. Dosunmu, R. P. Mirin, and M. S. Unlu, "Resonant cavity enhanced photodiodes with a broadened spectral peak," in Proceedings of the 14th Annual Meeting of the IEEE Lasers and Electro-Optics Society (LEOS '01), vol. 2, pp. 768-769, IEEE, San Diego, Calif, USA, November 2001.

[39] B. H. Na, G. W. Ju, H. J. Choi, Y. C. Cho, Y. H. Park, and Y. T. Lee, "Large aperture asymmetric Fabry Perot modulator based on asymmetric tandem quantum well for low voltage operation," Optics Express, vol. 20, no. 6, pp. 6003-6009, 2012.

[40] S. H. Lee, C. Y. Park, J.-W. You, H. Yoon, Y.-C. Cho, and Y.H. Park, "850 nm IR transmissive electro-absorption modulator using GaAs micromachining," Sensors and Actuators A: Physical, vol. 197, pp. 47-52, 2013.

[41] S. A. Alboon and R. G. Lindquist, "Flat top liquid crystal tunable filter using coupled Fabry-Perot cavities," Optics Express, vol. 16, no. 1, pp. 231-236, 2008.

[42] A. A. M. Saleh and J. Stone, "Two-stage Fabry-Perot filters as demultiplexers in optical FDMA LAN's," Journal of Lightwave Technology, vol. 7, no. 2, pp. 323-330, 1989.

[43] E. Dorjgotov, A. Bhowmik, and P. Bos, "Design of a wide bandwidth switchable mirror based on a liquid crystal etalon," Journal of Applied Physics, vol. 105, no. 10, Article ID 104906, 2009.

[44] Y. Zhong, Y. C. Shin, C. M. Kim et al., "Optical and electrical properties of indium tin oxide thin films with tilted and spiral microstructures prepared by oblique angle deposition," Journal of Materials Research, vol. 23, no. 9, pp. 2500-2505, 2008.

[45] K. Robbie and M. J. Brett, "Sculptured thin films and glancing angle deposition: growth mechanics and applications," Journal of Vacuum Science and Technology A: Vacuum, Surfaces and Films, vol. 15, no. 3, pp. 1460-1465, 1997.

[46] S. J. Jang, Y. M. Song, C. I. Yeo, C. Y. Park, and Y. T. Lee, "Highly tolerant a-Si distributed Bragg reflector fabricated by oblique angle deposition," Optical Materials Express, vol. 1, no. 3, pp. 451-457, 2011.

[47] M. C. Y. Huang, Y. Zhou, and C. J. Chang-Hasnain, "A surfaceemitting laser incorporating a high-index-contrast subwavelength grating," Nature Photonics, vol. 1, no. 2, pp. 119-122, 2007.

[48] C. Chase, Y. Zhou, and C. J. Chang-Hasnain, "Size effect of high contrast gratings in VCSELs," Optics Express, vol. 17, no. 26, pp. 24002-24007, 2009.

[49] W. Hofmann, C. Chase, M. Müller et al., "Long-wavelength high-contrast grating vertical-cavity surface-emitting laser," IEEE Photonics Journal, vol. 2, no. 3, pp. 415-422, 2010.

[50] P. Gilet, N. Olivier, P. Grosse et al., "High-index-contrast subwavelength grating VCSEL," in Vertical-Cavity Surface-Emitting Lasers XIV, vol. 7615 of Proceedings of SPIE, p. 76150J, San Francisco, Calif, USA, January 2010.

[51] S. Boutami, B. Benbakir, J.-L. Leclercq, and P. Viktorovitch, "Compact and polarization controlled $1.55 \mu \mathrm{m}$ vertical-cavity surface-emitting laser using single-layer photonic crystal mirror," Applied Physics Letters, vol. 91, no. 7, Article ID 071105, 2007.

[52] S. Boutami, B. Benbakir, X. Letartre, J. L. Leclercq, P. Regreny, and P. Viktorovitch, "Ultimate vertical Fabry-Perot cavity based on single-layer photonic crystal mirrors," Optics Express, vol. 15, no. 19, pp. 12443-12449, 2007.

[53] I.-S. Chung, J. Mørk, P. Gilet, and A. Chelnokov, "Subwavelength grating-mirror VCSEL with a thin oxide gap," IEEE Photonics Technology Letters, vol. 20, no. 2, pp. 105-107, 2008.

[54] M. C. Y. Huang, Y. Zhou, and C. J. Chang-Hasnain, "A nanoelectromechanical tunable laser," Nature Photonics, vol. 2, no. 3, pp. 180-184, 2008.

[55] I.-S. Chung, V. Iakovlev, A. Sirbu et al., "Broadband MEMStunable high-index-contrast subwavelength grating longwavelength VCSEL," IEEE Journal of Quantum Electronics, vol. 46, no. 9, pp. 1245-1253, 2010.

[56] Y. Zhou, M. C. Y. Huang, C. Chase et al., "High-index-contrast grating (HCG) and Its applications in optoelectronic devices," IEEE Journal on Selected Topics in Quantum Electronics, vol. 15, no. 5, pp. 1485-1499, 2009.

[57] W. Yang, T. Sun, Y. Rao et al., "High speed optical phased array using high contrast grating all-pass filters," Optics Express, vol. 22, no. 17, pp. 20038-20044, 2014.

[58] C. J. Chang-Hasnain, "High-contrast gratings as a new platform for integrated optoelectronics," Semiconductor Science and Technology, vol. 26, no. 1, Article ID 014043, 2011.

[59] C. J. Chang-Hasnain and W. Yang, "High-contrast gratings for integrated optoelectronics," Advances in Optics and Photonics, vol. 4, no. 3, pp. 379-440, 2012.

[60] C. Chang-Hasnain and W. Yang, "Integrated optics using high contrast gratings," in Photonics, D. L. Andrews, Ed., chapter 2, pp. 57-105, John Wiley \& Sons, 2015.

[61] B. H. Na, G. W. Ju, H. J. Choi et al., "Wide spectral bandwidth electro-absorption modulator using coupled micro-cavity with asymmetric tandem quantum well," Optics Express, vol. 20, no. 17, pp. 19511-19519, 2012. 

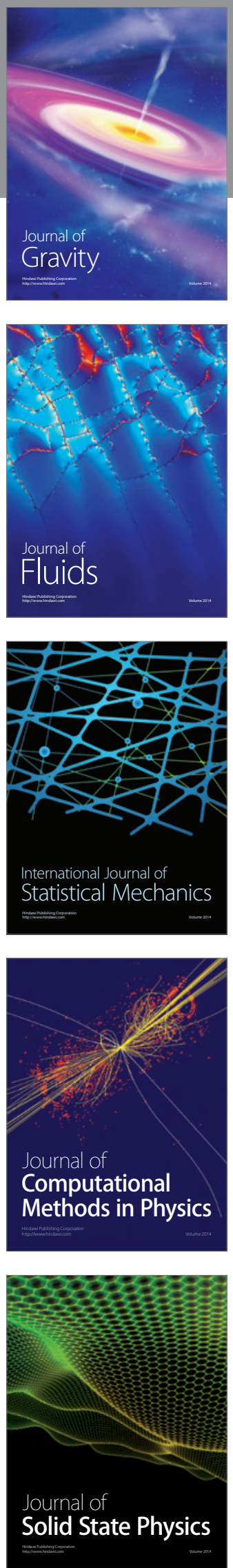

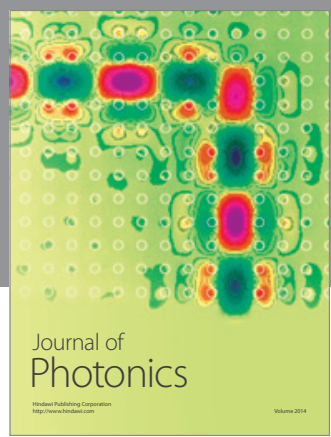

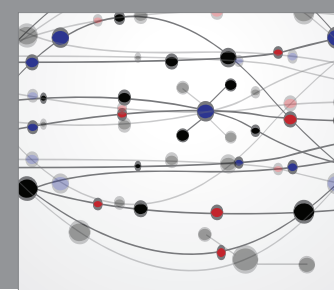

The Scientific World Journal

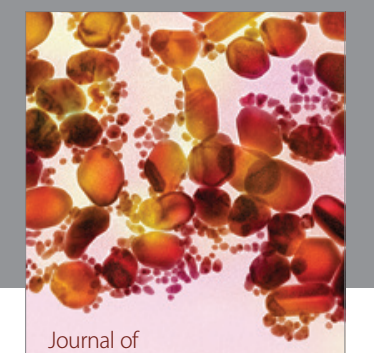

Soft Matter
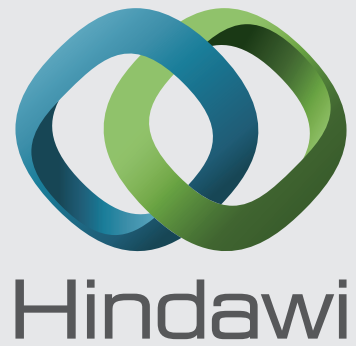

Submit your manuscripts at

http://www.hindawi.com
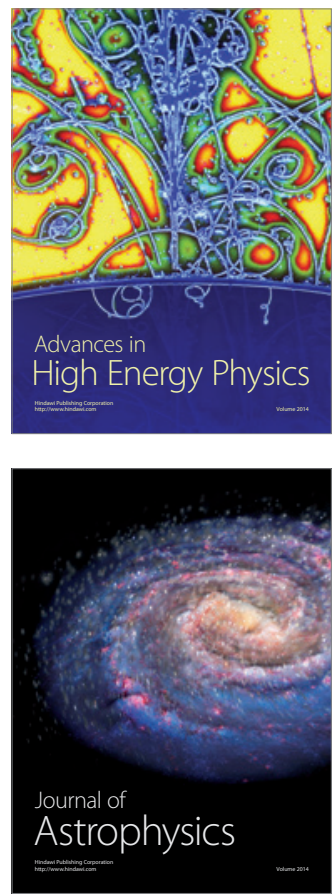
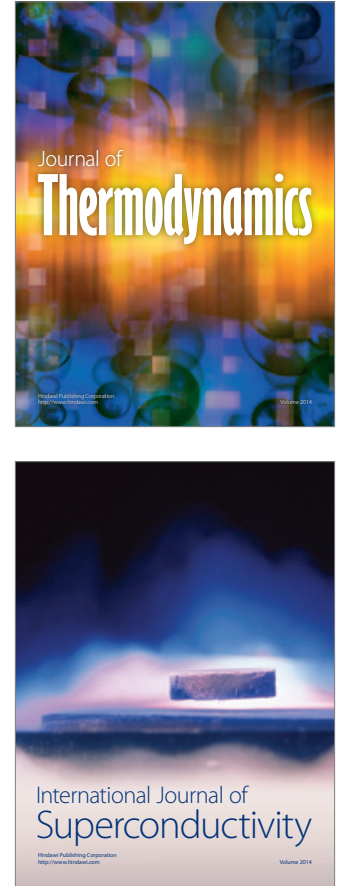
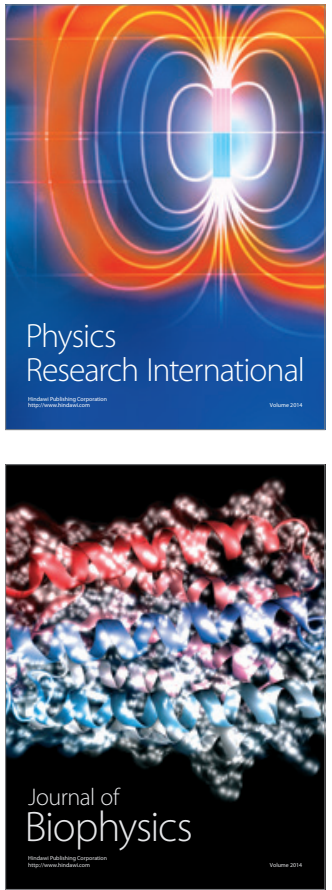
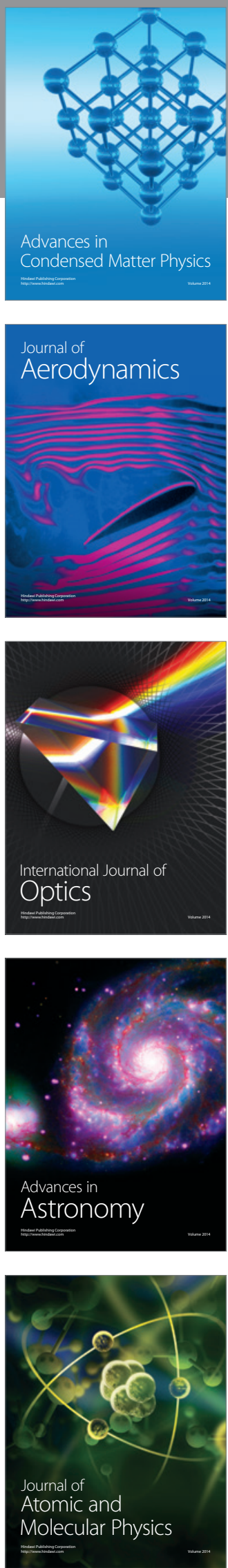